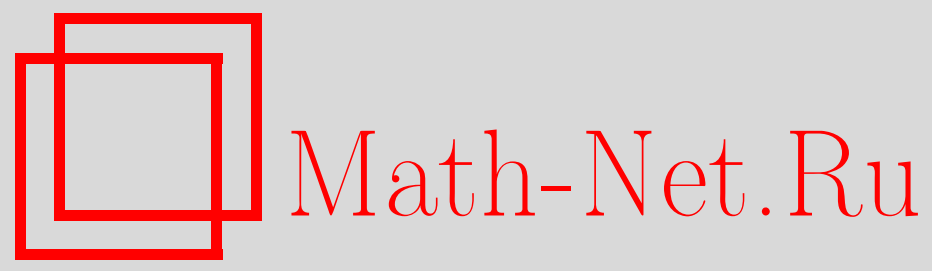

Е. С. Корчак, Математическое моделирование как основа создания системы эффективного торможения подвижной поперечины гидравлического пресса в верхнем положении, Вестн. Сам. гос. техн. ун-та. Сер. Физ.-мат. науки, 2012, выпуск 1(), 192-197

DOI: https://doi.org/10.14498/vsgtu917

Использование Общероссийского математического портала Math-Net.Ru подразумевает, что вы прочитали и согласны с пользовательским соглашением

http: //www . mathnet.ru/rus/agreement

Параметры загрузки:

IP : 44.207 .124 .84

26 апреля 2023 г., 14:14:05

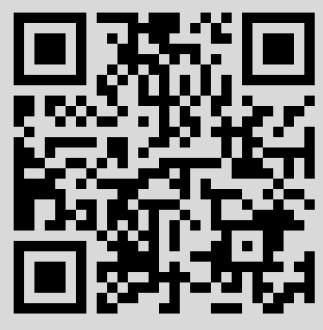




\title{
МАТЕМАТИЧЕСКОЕ МОДЕЛИРОВАНИЕ КАК ОСНОВА СОЗДАНИЯ СИСТЕМЫ ЭФФЕКТИВНОГО ТОРМОЖЕНИЯ ПОДВИЖНОЙ ПОПЕРЕЧИНЫ ГИДРАВЛИЧЕСКОГО ПРЕССА В ВЕРХНЕМ ПОЛОЖЕНИИ
}

\section{E. C. Корчак}

Донбасская государственная машиностроительная академия, 84313, Украина, Краматорск, ул. Шкадинова, 72.

E-mail: helen_korchak@ukr.net

\begin{abstract}
Рассмотрена одномассовая математическая модель торможения подвижной поперечины гидравлического пресса в верхнем положении. Проанализировано влияние времени закрытия регулирующего клапана, его конструктивной характеристики и коэффициента качества гидросистемы на динамику торможения. Даны практические рекомендации по созданию систем эббективного торможения подвижной поперечины гидравлического пресса в верхнем положении.
\end{abstract}

Ключевые слова: гидравлический пресс, торможение, клапан, конструктивная характеристика, подвижная поперечина.

Введение. Наиболее распространенным способом торможения гидропривода является изменение сопротивления соответствующих магистралей [1]. При этом сам процесс торможения может осуществляться тремя способами:

- установкой тормозного устройства в напорную магистраль;

- установкой тормозного устройства в сливную магистраль;

- комбинацией первых двух способов.

Однако независимо от способа осуществления торможения подвижных частей действие тормозных устройств основано на двух принципах:

- уменьшение количества энергии, подводимой жидкостью к исполнительному органу, что достигается путем изменения характеристик источника энергии, изменения эффективной площади исполнительного органа, включения тормозного устройства в напорную магистраль;

- увеличение силы сопротивления, что достигается путем увеличения сил сопротивления со стороны специально установленного тормозного устройства либо при его включении в сливную магистраль.

1. Анализ результатов математического моделирования процесса торможения двухмассовой системы. Как известно, торможение подвижной поперечины в верхнем положении осуществляется путем закрытия впускного клапана возвратных цилиндров, чем достигается снижение активной силы подъема и поперечина замедляет свое движение [2]. Тормозное устройство (закрывающийся впускной клапан) установлено в напорной магистрали. Жидкость в магистрали «аккумулятор - пресс» и металлические подвижные части, двигавшиеся до закрытия клапана как одна масса, приобретают характер двухмассовой системы. При этом разделителем масс служит закрывающийся регулирующий клапан. Поэтому при составлении математической модели

Елена Сергеевна Корчак (к.т.н.), доцент, каф. машин и технологии обработки металлов давлением. 
торможения подвижных частей в качестве одной из масс двухмассовой системы приняли приведенную к поперечине массу жидкости на участке «аккумулятор - регулирующий клапан», а второй - сумму масс металлических подвижных частей и жидкости на участке «регулирующий клапан - пресс».

Численным анализом математической модели торможения подвижной поперечины гидравлического пресса в верхнем положении на базе описанной двухмассовой системы установлено, что регулирующие свойства у впускного клапана возвратных цилиндров практически отсутствуют, из-за чего даже при самых благоприятных режимах торможения выбег поперечины вверх, приводящий к вакуумированию системы возвратных цилиндров, неизбежен. При этом торможение подвижной поперечины сопровождается гидроударами различной интенсивности независимо от вида конструктивной характеристики регулирующего клапана и времени его закрытия, а также величины коэффициента $\alpha$ - коэффициента качества гидравлической системы, т. е. отношения гидравлического сопротивления регулирующего клапана к общему сопротивлению магистрали «аккумулятор - пресс». Проведенный анализ также показал, что увеличение времени торможения свыше 0,2 с позволяет снизить удары, однако затяжное торможение приводит к снижению производительности пресса, что является недопустимым [3].

Таким образом, результаты математического моделирования процесса торможения двухмассовой системы позволяют сделать вывод, что существующий способ торможения подвижной поперечины в верхнем положении является неэффективным. Для обеспечения быстрого и безударного торможения необходимо разработать новый механизм осуществления этого процесса.

\section{2. Описание математической модели торможения одномассовой системы.} При торможении подвижных частей гидропривода должны быть исключены условия, способствующие возникновению колебаний и ударов, а также динамических перегрузок элементов системы. Следовательно, модуль ускорения подвижных частей должен быть ограничен.

При установке тормозного устройства в напорную магистраль возможны вакуумирование гидросистемы и разрыв сплошности потока жидкости. Это ограничивает максимально достижимый перепад давления в тормозном устройстве, а следовательно, и максимально достижимый модуль ускорения.

Когда же тормозное устройство включено в сливную магистраль, величина модуля ускорения ограничена из-за сжимаемости жидкости. В этом случае достижимо практически любое необходимое описание закона торможения, а для анализа динамики торможения поперечины принимается одномассовая «жёстко-упругая» модель гидропривода. Жидкость в приводе и металлические подвижные части движутся как одна приведенная к поперечине масса. При этом за основу математического описания процесса торможения принимается следующее уравнение [3]:

$$
a \frac{d^{2} S}{d t^{2}}+b\left[1+\alpha\left(\frac{1}{\left(1-t / t_{3}\right)^{2 n}}-1\right)\right]\left(\frac{d S}{d t}\right)^{2}-c-k_{T} S=0
$$

где $a$-приведенные к поперечине движущиеся массы; $c$-активная сила, действующая на приведенные массы; $b$ - коэффициент гидравлического сопротивления магистрали «аккумулятор - пресс» ; $S$ - путь, пройденный приведенной массой при торможении; $t_{3}$ - время закрытия регулирующего кла- 
пана; $k_{T}$ - приведенная линейная жесткость упругих элементов гидропривода; $n$ - показатель вида конструктивной характеристики регулирующего клапана; $\alpha$ - коэффициент качества гидросистемы.

Член в квадратных скобках уравнения (1) представляет собой коэффициент, учитывающий прирост гидравлического сопротивления регулирующего клапана в процессе его закрытия. Коэффициент $\alpha$ указывает на степень искажения скоростной характеристики клапана под влиянием гидравлического сопротивления магистрали «аккумулятор - пресс».

\section{3. Аналитическое исследование математической модели торможения одно-} массовой системы. Как видно из уравнения (1), существенное влияние на динамику торможения подвижной поперечины в верхнем положении в условиях одномассовой системы оказывают следующие параметры: $t_{3}, n$ и $\alpha$. Проведем аналитическое исследование влияния каждого из них на примере ковочного гидравлического пресса усилием $60 \mathrm{MH}$, соответствующие параметры которого, входящие в уравнение (1), приведены в таблице.

\section{Параметры уравнения (1)}

\begin{tabular}{c|c|c}
\hline Параметр & Размерность & Величина \\
\hline$a$ & $\mathrm{kг}$ & $2,5 \cdot 10^{6}$ \\
$b$ & $\mathrm{Kг} / \mathrm{м}$ & $6,4 \cdot 10^{7}$ \\
$c$ & $\mathrm{H}$ & $2,6 \cdot 10^{6}$ \\
$\alpha$ & - & 0,7 \\
$k_{T}$ & $\mathrm{H} / \mathrm{M}$ & $8,4 \cdot 10^{7}$ \\
\hline
\end{tabular}

Уравнение (1) аналитически не решается. Для его решения использовали численный метод РунгеКутта. В соответствии с результатами решения, представленными на рис. 1, можно отметить, что различия в динамике торможения клапанами с различными конструктивными характеристиками проявляются при всех значениях времени закрытия. Тормозное устройство - регулирующий клапан - с релейной (a), линейной (б) и квадратичной (в) конструктивными характеристиками закрывали за 0,01, 0,1 и 0,2 с.

При торможении релейным клапаном (рис. 1, а) со временем закрытия 0,01 с резкое снижение скорости $V$ наблюдается только в конце закрытия в течение последних 0,0035 с, а ускорение $J$ достигает пикового значения $74 \mathrm{~m} / \mathrm{c}^{2}$.

При торможении линейным клапаном (рис. 1, б) снижение скорости $V$ начинается гораздо раньше, а пиковое значение ускорения $J$ составляет $62 \mathrm{~m} / \mathrm{c}^{2}$.

Торможение квадратичным клапаном (рис. 1, в) за 0,01 с характеризуется снижением скорости $V$ почти с самого начала закрытия, причем кривая $V(t)$ приходит к нулевому значению с минимальной крутизной. Пиковое значение ускорения $J$ наблюдается на середине хода клапана и равно $48 \mathrm{~m} / \mathrm{c}^{2}$.

Путь торможения для всех клапанов составил порядка 3 мм.

С увеличением времени закрытия клапана его влияние на динамику торможения усиливается. Так, при $t_{3}=0,1$ с снижение скорости $V$ осуществляется релейным клапаном в течение 0,06 с, линейным - 0,08 c, квадратичным - в течение всего времени закрытия. Наибольшее значение ускорения $J$ наблюдается у релейного клапана $20 \mathrm{~m} / c^{2}$, а ускорения у линейного и квадратичного клапанов составляют $7 \mathrm{~m} / \mathrm{c}^{2}$ и $4 \mathrm{~m} / \mathrm{c}^{2}$ соответственно. Причем для последнего случая характерно наличие пика ускорения примерно на середине хода, а к концу закрытия величина $J$ стремится к нулю. При этом путь торможения составил: для релейного клапана - 18 мм, для линейного - 14 мм, для квадратичного - 12 мм. 

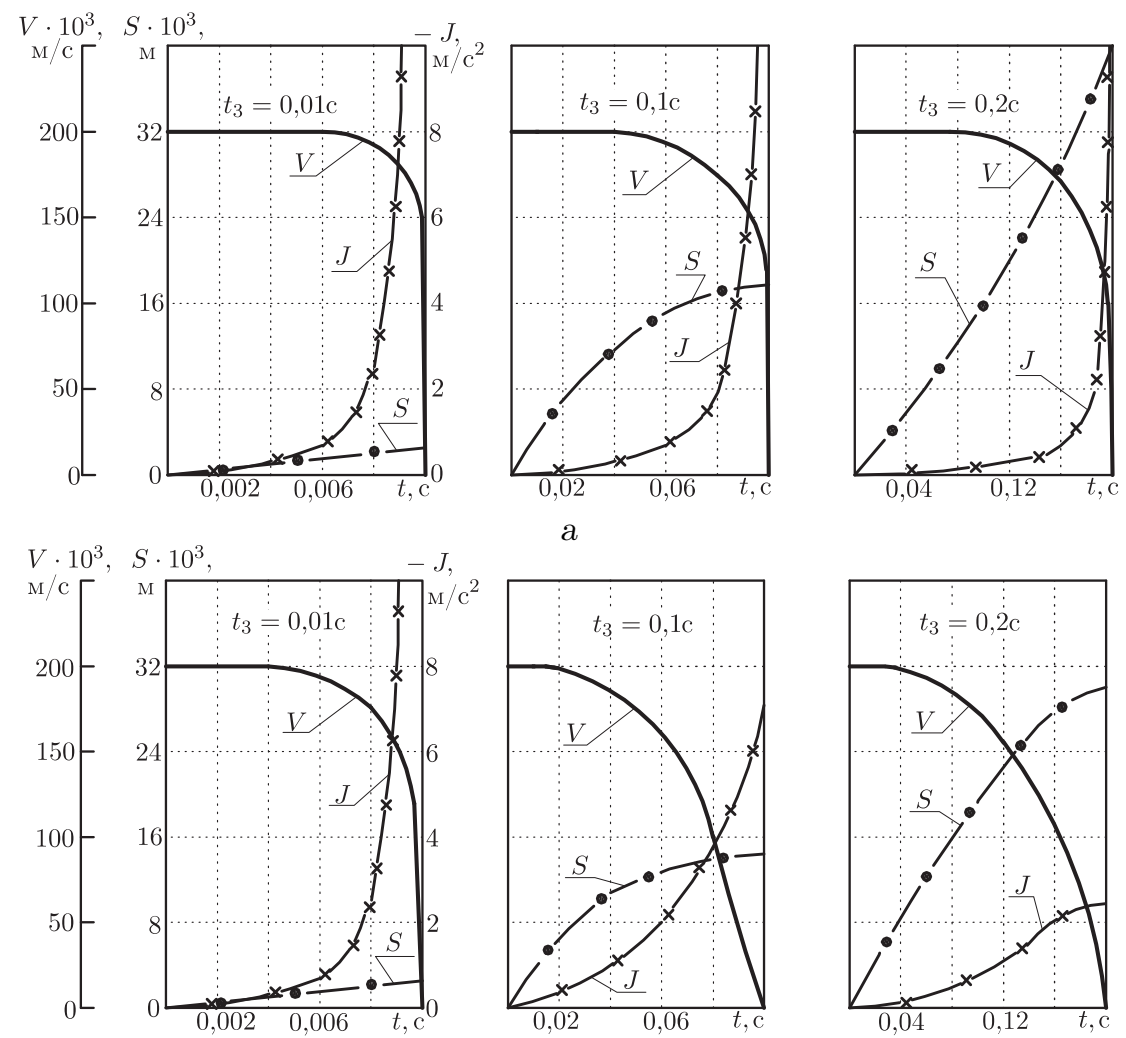

a
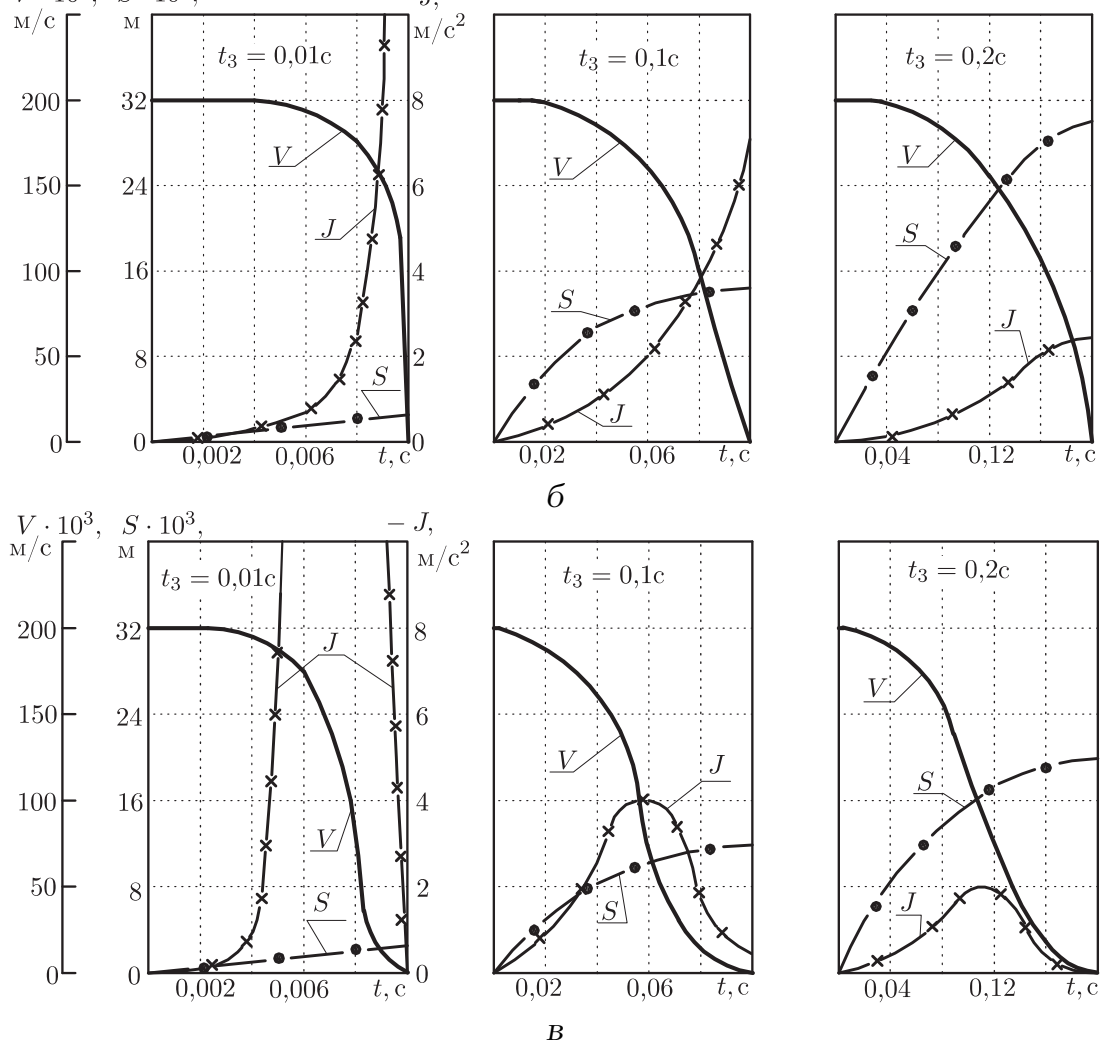

Рис. 1. Влияние регулирующего клапана с релейной (a), линейной (б) и квадратичной (в) конструктивными характеристиками на динамику торможения подвижной поперечины

При $t_{3}=0,2$ с все вышеописанные особенности сохраняются. При этом снижение скорости $V$ осуществляется более плавно. Максимальное значение ускорения $J$ наблюдается у релейного клапана $10 \mathrm{~m} / \mathrm{c}^{2}$, а ускорения у линейного и квадратичного клапанов составляют $2,2 \mathrm{M} / \mathrm{c}^{2}$ и $2,0 \mathrm{~m} / \mathrm{c}^{2}$ соответственно. При этом путь торможения составил: для релейного клапана - 40 мм, для линейного - 30 мм, для квадратичного - 20 мм.

Проанализируем влияние коэффициента $\alpha$ на динамику торможения поперечины (рис. 2) при времени закрытия клапана $t_{3}=0,3$ с. 


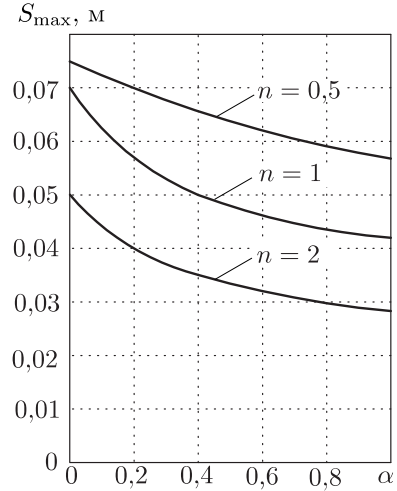

a

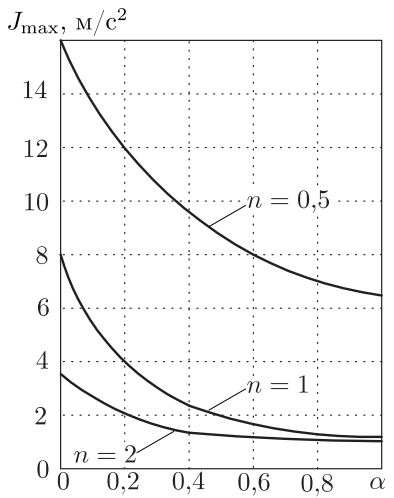

б

Из рис. 2, а видно, что чем больше значение коэффициента $\alpha$, тем меньше величина максимального пути торможения $S_{\max }$ поперечины. При $\alpha$, близком к единице, для релейного клапана этот параметр составил 0,057 м, для линейного - 0,042 м, для квадратичного 0,028 м.

Рис. 2, б показыва-

Рис. 2. Изменения максимальных пути торможения $S_{\max }\left(\right.$ а) и ускорения $J_{\max }(б)$ поперечины от коэффициента $\alpha$ и конструктивной характеристики $n$ регулирующего клапана

ет, что чем больше значение коэффициента $\alpha$, тем меньше значение ускорения $J_{\max }$ при торможении.

Каким бы ни был коэффициент $\alpha$, при торможении релейным клапаном имеет место интенсивный гидроудар, о чем свидетельствует значение $J_{\max }=$ $=6,5 \mathrm{~m} / \mathrm{c}^{2}$ при $\alpha \approx 1$. При торможении линейным и квадратичным клапанами максимальное значение ускорения $J_{\max }$ при $\alpha=0,95$ составляет $1,25 \mathrm{~m} / \mathrm{c}^{2}$ и $1,2 \mathrm{M} / \mathrm{c}^{2}$ соответственно.

Таким образом, проведенный анализ показал, что когда тормозное устройство включено в сливную магистраль, обеспечивается четкое без выбегов и вакуумирования торможение подвижной поперечины в верхнем положении без колебательных явлений и гидроударов. Для осуществления такого торможения клапан с релейной конструктивной характеристикой совершенно неприменим, так как даже при увеличении времени его закрытия интенсивный гидравлический удар неминуем. Клапан же с линейной и квадратичной конструктивными характеристиками, наоборот, создает условия для безударного торможения поперечины при условии, что время закрытия составит не менее 0,3 с, а коэффициент $\alpha$ - не менее 0,9 .

Заключение. Выполненное аналитическое исследование одномассовой математической модели позволяет сформулировать следующие основные рекомендации по созданию систем эффективного торможения подвижной поперечины гидравлического пресса в верхнем положении.

На основе проведенных в работе исследований привод ковочного гидравлического пресса может быть усовершенствован следующим образом: торможение подвижной поперечины в верхнем положении необходимо осуществлять путем закрытия наполнительно-сливного клапана (HCK) с дополнительным дросселирующим элементом. Этим обеспечивается четкое торможение поперечины в крайнем верхнем или в любом промежуточном положении без колебательных явлений, гидроударов и вакуумирования. Это достигается тем, что HCK снабжен индивидуальным следящим сервоприводом и дополнительным дроссельно-регулирующим клапаном, которые вместе образуют наполнительно-сливной блок наполнения и слива рабочей жидкости и торможения поперечины в верхнем положении.

Независимость работы НCK от давления в подводящем трубопроводе воз- 
вратных цилиндров, а также установка дополнительного дроссельно-регулирующего клапана с индивидуальным следящим сервоприводом обеспечивают торможение поперечины в верхнем положении без колебательных явлений и гидроударов, уменьшение машинного времени, повышение продуктивности пресса, его надёжности и долговечности.

\title{
БИБЛИОГРАФИЧЕСКИЙ СПИСОК
}

1. Левитский Н.И., Цуханова Е.А Расчёт управляющих устройств для торможения гидроприводов/ ред. В.И. Молчанова. М.: Машиностроение, 1971.232 с. [Levitskiy N.I., Tsukhanova E.A. Calculation of control devices for hydraulic drives inhibition / ed. V. I. Molchanova. Moscow: Mashnostroenie, 1971. 232 pp.]

2. Шинкаренко О.М., Корчак, E.C. Торможение подвижных частей ковочных прессов с насосно-аккумуляторным приводом // Извест. ТулГУ. Сер. Механика дебормируемого твердого тела и обработка металлов давлением, 2006. №1. С. 346-355. [Shinkarenko O.M., Korchak S. E. Inhibition of moving parts of forging presses with pump battery drive// Izvest. TulGU. Ser. Mekhanika deformiruemogo tverdogo tela i obrabotka metallov davleniem, 2006. no. 1. Рp. 346-355].

3. Корчак E. C. Анализ математической модели торможения подвижной поперечины гидравлического пресса в верхнем положении // Becmн. Сам. гос. техн. ун-та. Сер. Физ.мат. наукu, 2009. №2(19). C. 153-159. [Korchak E.S. Analysis of mathematical model of hydraulic press ram braking in upper position // Vestn. Samar. Gos. Tekhn. Univ. Ser. Fiz.-Mat. Nauki, 2009. no. 2(19). Pp. 153-159].

Поступила в редакцию 25/I/2011;

в окончательном варианте - 06/XI/2011.

MSC: 93A30

\section{MATHEMATICAL MODELING AS A BASIS FOR CREATION OF THE EFFECTIVE BRAKING SYSTEM OF HYDRAULIC PRESS RAM IN UPPER POSITION}

\author{
E. S. Korchak \\ Donbass State Engineering Academy, \\ Kramatorsk, Ukraine \\ E-mail: helen_korchak@ukr.net
}

Single-mass mathematical model of hydraulic press ram braking in upper position is considered. The influence of control valve closing time, its constructive characteristics and coefficient of hydraulic system quality on dynamics of ram braking are analyzed. Practical recommendations on creating effective braking systems of hydraulic press ram in upper position are given.

Key words: hydraulic press, braking, valve, constructive characteristic, ram.

Original article submitted 25/I/2011; revision submitted 06/XI/2011.

Elena S. Korchak (Ph.D. (Tech.)), Associate Professor, Dept. of Machinery \& Metalforming Technology. 